Brief note

\title{
KINEMATIC ANALYSIS OF CPM MACHINE SUPPORTING TO REHABILITATION PROCESS AFTER SURGICAL KNEE ARTHROSCOPY AND ARTHROPLASTY
}

\author{
R. TROCHIMCZUK* and T. KUŹMIEROWSKI \\ Department of Automatics and Robotics \\ Faculty of Mechanical Engineering \\ Bialystok University of Technology \\ Ul. Wiejska 45C, 15-351 Białystok, POLAND \\ E-mails: r.trochimczuk@pb.edu.pl; \\ t.kuzmierowski@pb.edu.pl
}

\begin{abstract}
Existing commercial solutions of the CPM (Continuous Passive Motion) machines are described in the paper. Based on the analysis of existing solutions we present our conceptual solution to support the process of rehabilitation of the knee joint which is necessary after arthroscopic surgery. For a given novel structure we analyze and present proprietary algorithms and the computer application to simulate the operation of our PCM device. In addition, we suggest directions for further research.
\end{abstract}

Key words: rehabilitation, CPM machine, kinematic analysis, arthroscopy, arthroplasty, four bar linkage mechanism, crank-slider mechanism.

\section{Introduction}

Modern society is increasingly vulnerable to all kinds of ailments related to the human musculoskeletal system. The most common are injuries and diseases that affect the largest human joint - the knee joint. The may be caused by the following: overloaded-degenerative changes that result from the aging of the human body, injuries and microtrauma experienced during lifetime (e.g., small injuries stemming from intensive sport activities that extremely overloaded the knee joint such as football, skiing or skating), unsuitable lifestyle (e.g., obesity, sitting job), pathological changes in the knee joint (e.g., changes resulting from pharmacological treatment or diseases such as diabetes), birth defects.

Whatever the cause of problems with the knee joint, the pain and limitation of joint motion of the patient to a great extent eliminate them from a normal everyday activity and professional life. With a significant severity of symptoms this can lead to muscle atrophy, reduction or even cessation of normal everyday functioning. In extreme cases, a patient may end up on an operating table, where the surgents perform a total joint replacement (knee arthroplasty) or replace the damaged parts of the joint with prosthesis (implant). As for as the surgical procedures on the knee joint are concerned, arthroscopy, which is considered to be minimally invasive, is relatively often performed.

This method is quite widely used since the 1980s. It allows medical diagnosis (e.g., endoscopy of the inside of the joint) as well as advanced procedures, such as surgical resections or reproductive surgeries. The former involve the removal of the damaged parts of the joints, the latter is performed to replace the damaged parts with a new joint. Typically, the need for treatment includes reconstruction of the cruciate

\footnotetext{
* To whom correspondence should be addressed
} 
ligament and collateral, treatment of damaged meniscus, surgery degenerative changes, treatment of instability patellar.

Regardless of the adopted method of treatment or diagnosis of the knee, it is necessary after completed surgery procedures, that the patient makes a recovery and regains mobility in the range of daily locomotion. In some cases, also comes into play recovery of the proper muscle mass to carry out the full range of motion in a joint. To achieve this different methods of rehabilitation are used. Part of the normal practice is supported by a device providing continuous passive motion (Continuous Passive Motion Machine - CPM machine). The machine allows flexion and extension of the knee at a specified range of angle. More detailed description of this method can be found in the references.

In this paper the authors present a review of the existing commercial solutions of CPM machine and define engineering guidelines necessary to consider before developing their own novel concept of a device for rehabilitation. The kinematic analysis and the original software for simulating the work (motion, position, velocity, acceleration) of the proposed structure of mechanism of the new CPM machine the authors present in this work. The described software generates results that can be used in future studies on the dynamics of this mechanism.

\section{An overview of existing solution of CPM devices}

In many medical centers as early as in the first hours, days, immediately after initial surgery, CPM machines are becoming widely used for rehabilitation. They are used not only to assist in the knee-joint rehabilitation process, but also in the other joint rehabilitation, i.e., the hip-joint, the elbow-joint, the shoulder-joint, the wrist, the fingers and the feet joints or the stimulation of the muscles around the mouth and the lower jaw. Obviously, the structure of the CPM machines differs depending on use. Currently on the market we have a large number of different solutions and their structures may vary from each other depending on the manufacturer providing the devices. For the purpose of this study, the authors describe solutions that are usually offered by rental companies that specialize in rehabilitation equipment and operate in Poland. Among them are the product of ARTROMOT family (Fig.1a), Chattanooga OptiFlex3 CPM machine (Fig.1b) and Jace K200 The Zero Machine CPM machine provided by Jace System (Fig.1c). Description of other examples of CPM machine solutions can be found, inter alia, in the works (Ortega et al., 2012; Erhan et al., 2009; Riidiger et al., 2009).

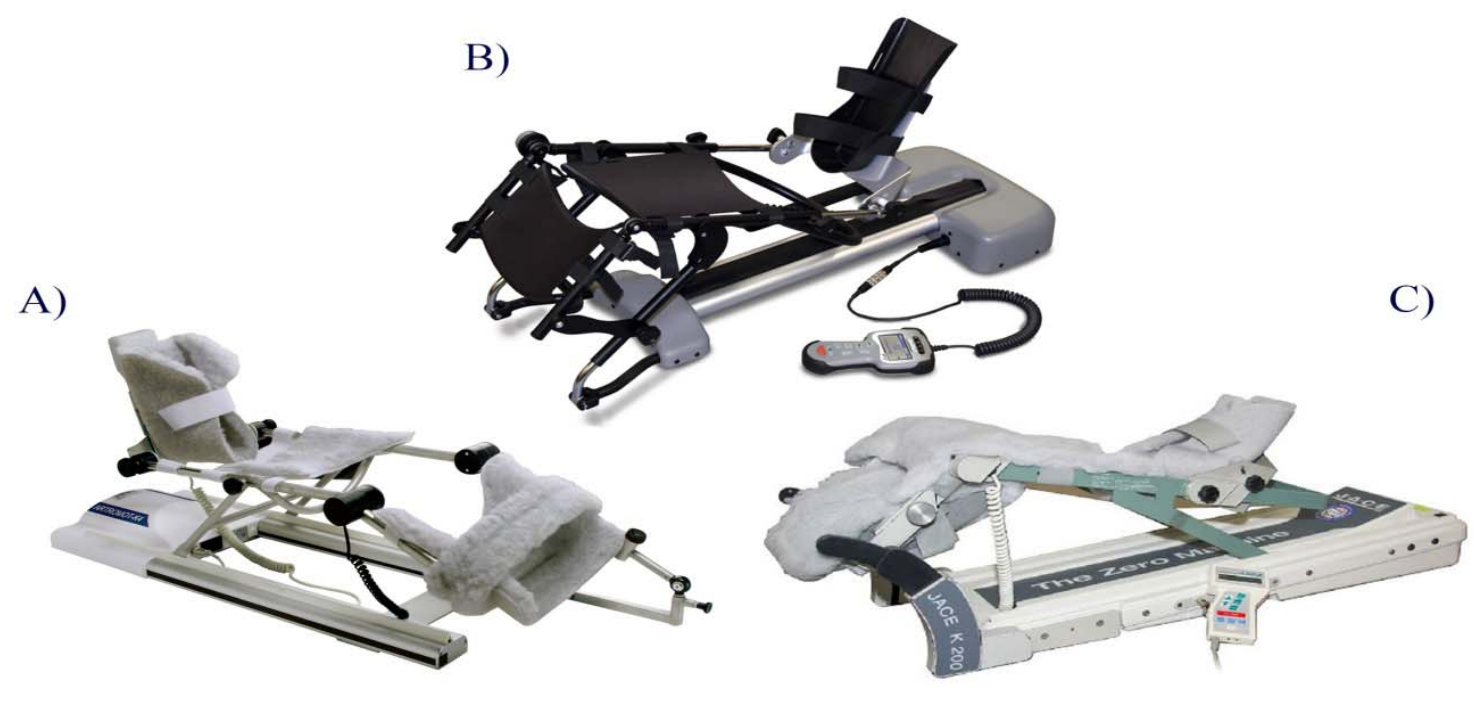

Fig.1. View of commercial examples of CPM machines (ArtroMed Kraków, 2014; Hunter Distributing, 2014) 
Analyzing the technical specifications of CPM machines and their mechanical structures, the weight of each of the described systems can be determined to be in the range of about $10 \mathrm{~kg}$. These are usually mobile solutions. CPM machines can adapt to patients from 120 to $200 \mathrm{~cm}$ tall and weighing up to $160 \mathrm{~kg}$. Therefore, they provide the possibility of adjusting according to the individual biophysical characteristics of the patient. Typically the motion of joints of the CPM machine is provided by an electric linear actuator or by a spindle-nut system with a DC motor. The angle of the of the motion varies from $-10^{0}$ to $125^{\circ}$. The measurement of the angle of deflection is implemented using a potentiometric system, which is located in the rotary joint mechanism. The CPM machine is typically controlled via the operator panel connected by wire to the rehabilitation device. It allows current to operate the unit in terms of setting the range of oscillation and the progress of automatic programming exercises and their duration in each of these cases. Systems are also capable of increasing/decreasing the velocity of movement and the amount of force exerted on the limb of a patient taking into account the individual post-operative pain tolerance.

Some of these solutions use the four-bar linkage mechanism to enhance the stability and rigidity of movement in the mechanical structure. The elements of the mechanism of the CPM machine are usually tubes or flats, made of lightweight and durable aluminum alloys. Additionally, the devices are equipped with components that increase comfort level of the limb support and as well as fixing and stabilizing strips which can be used in further stages of the rehabilitation process.

\section{Engineering aspects of the design of CPM machines}

When designing a solution of a CPM machine one should be aware that depending on current needs, the device can provide different additional functionality beyond ensuring the proper movement of the rehabilitation limb. Hence, analyzing the solutions that are currently on the market or specialized solutions used in medical facilities, you can come to the conclusion: despite a large number of solutions the optimal device is yet to be created. Manufacturers of rehabilitation systems in order to differentiate their own products from other products available on the market, provide specific additional functionality (e.g., extended user interface, storing the number of cycles, etc.). However, in most cases it does not significantly affect the rehabilitation process and is essentially important from the marketing point of view.

To take up a challenge of creating a proprietary solution of a CPM machine you have to consider the following engineering aspects (Wilk et al., 2004):

- adjustability to a wide range of patient sizes;

- comfort, particularly for long uninterrupted application;

- use a variety of settings: in bed while sleeping, while ambulating during the day, at home or in hospital;

- full anatomic range of motion capability and multi-axis motion capability;

- speed control and simple, straightforward operation;

- protection for unstable joints;

- durability and lightweight;

- low cost simple solution.

These features, however, do not indicate the direction of the mechanical development of the structure. There is no mention of the kinematics and dynamics of the system and how to control the rehabilitation system. An important engineering issue to consider is primarily a proper adjustment of the motion of the rehabilitation device to the possible movement of the lower limb. It is a very difficult issue because even though the regular range of movement of the knee is known (from $-10^{0}$ to $155^{0}$ ) (Ortega et al., 2012), the CPM machine may perform this movement in a different range. It is difficult to directly translate the axial movement of the mechanism into the same anatomical motion of the knee, which at a time can have, for example postoperative contracture. Hence, a large number of rehabilitation devices can only "approximate" normal motion of the knee hinge and the errors compensation of angular displacement of the devices elements are considered.

Mechanical components of the CPM machine should be rigid and work stable under maximum load (min. $110 \mathrm{~kg}$ or greater for adult men). Materials from which the device is to be made must be lightweight 
(commercial solutions weigh about $10 \mathrm{~kg}$ ). The mobile CPM device should meet all safety standards - this is a very important factor, because the patients operate the system by themselves at home.

Actuators used for the displacement of elements of the CPM machine should have a low power consumption and give the opportunity to change the velocity of movements. Nowadays, these should normally be integrated with mechanical transmissions. Actuators should work in a range of up to $50 \%$ of nominal load, in order to prolong their life.

Considering the standard load in one year a CPM machine may operate up to 6500 hours (over 3 million cycles). Most commercial available CPM machines are built for a service life of 5000 hours and a few are serviceable up to 15,000 hours (Saringer, 1993).

\section{Kinematic analysis of own concept of CPM machine}

Figure 2 shows the proposed novel structure of the Continuous Passive Motion device. It is a combined mechanism. It includes the four bar linkage mechanism (links 1, 2, 3) and crank-slider mechanism (links 3, 4, 5). Link 1 is the driven link. It rotates about point A. Additionally, it is possible to change the position of point $A$ along the $X$ axis. Changing the position of point A during the work of the mechanism influences the change of value of maximum deflection link 3 . Element 0 is a stationary base of the structure.

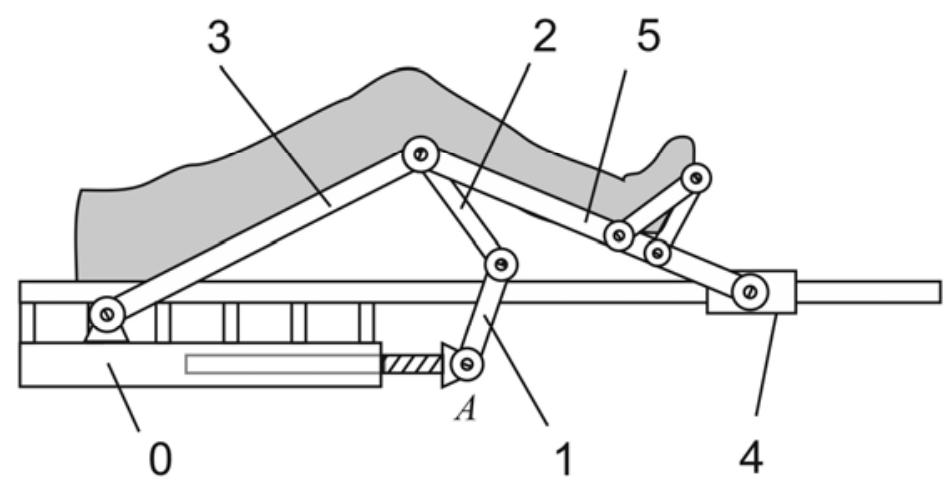

Fig.2. Proposed structure of own CPM machine.

The solution to the simple kinematics task consists in determining the angular position of the links 3 and 5 as a function of the angular position of the drive link 1 and the position of point A. The figure 3 shows the adopted angles designation and geometric position of points of the construction in the Cartesian coordinate system $X Y$.

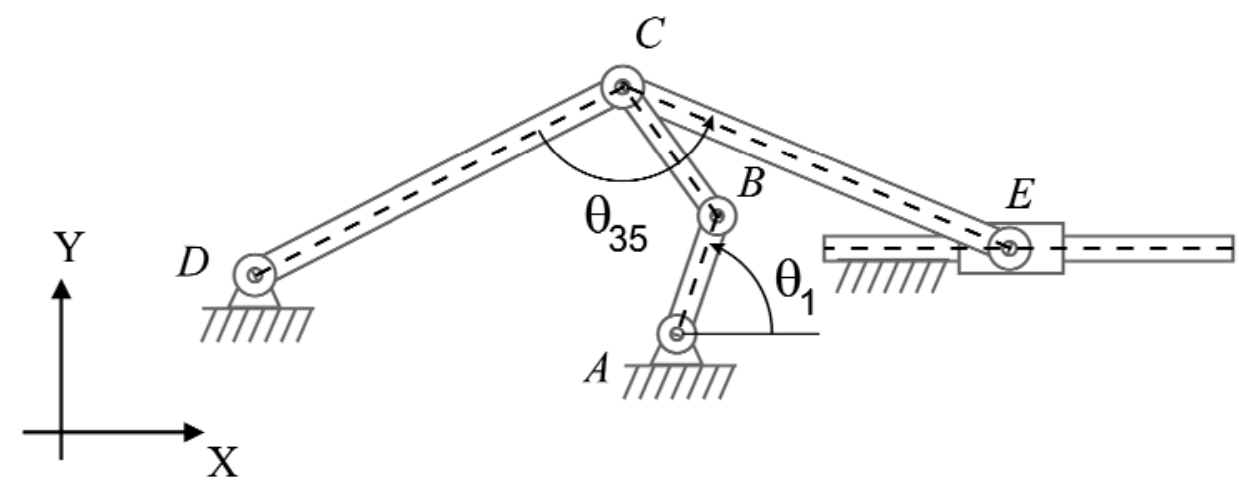

Fig.3. The adopted angles designation and position of points. 
The length of the links $l_{1}, l_{2}, l_{3}, l_{5}$ and $Y$-coordinate of the position of point $E$ (link 4 ) are the geometrical parameters.

A mathematical formula for the length of the links can be described by Eqs (4.1), (4.2), (4.3) and (4.4).

$$
\begin{aligned}
& \left(x_{B}-x_{A}\right)^{2}+\left(y_{B}-y_{A}\right)^{2}=l_{l}^{2}, \\
& \left(x_{C}-x_{B}\right)^{2}+\left(y_{C}-y_{B}\right)^{2}=l_{2}^{2}, \\
& \left(x_{C}-x_{D}\right)^{2}+\left(y_{C}-y_{D}\right)^{2}=l_{3}^{2}, \\
& \left(x_{C}-x_{E}\right)^{2}+\left(y_{C}-y_{E}\right)^{2}=l_{5}^{2},
\end{aligned}
$$

The solution boils down to determining the coordinates of points $B, C$, and $E$ at selected points $A, D$, and angle $\theta_{l}$.

The angle $\theta_{l}$ (the angle of the drive link) is a configuration parameter. The value of this angle uniquely determines the position of point $B$. The coordinates of point $\mathrm{B}$ can be determined from the system of Eqs (4.5).

$$
\begin{aligned}
& x_{B}=l_{1} \sin \theta_{1}, \\
& y_{B}=l_{1} \cos \theta_{1}
\end{aligned}
$$

Assuming that $x_{B} \neq x_{D}$ coordinates $X$ and $Y$ of point $C$ determined from Eqs (4.2) and (4.3) take the form Eqs (4.6).

$$
\begin{gathered}
y_{C}=\frac{-\left(x_{D} K_{2}-K_{1} K_{2}-y_{D}\right) \pm \sqrt{\left(x_{D} K_{2}-K_{1} K_{2}-y_{D}\right)^{2}-\left(K_{2}^{2}+1\right)\left(K_{1}^{2}-2 x_{D} K_{1}+y_{D}^{2}-l_{3}^{2}\right)}}{K_{2}^{2}+1}, \\
x_{C}=K_{1}-K_{2} y_{C}
\end{gathered}
$$

where

$$
\begin{aligned}
& K_{1}=\frac{x_{B}^{2}-x_{D}^{2}+y_{B}^{2}-y_{D}^{2}+l_{3}^{2}-l_{2}^{2}}{2\left(x_{B}-x_{D}\right)}, \\
& K_{2}=\frac{y_{B}-y_{D}}{x_{B}-x_{D}}
\end{aligned}
$$

We choose a higher value of the two possible solutions $y_{c}$.

With the known value $y_{e}$ coordinate $x_{e}$ determined from Eq.(4.4) takes the form Eq.(4.7). 


$$
x_{E}=x_{C} \pm \sqrt{2 x_{C}^{2}-\left(y_{C}-y_{E}\right)^{2}-l_{5}^{2}}
$$

We opt for a higher value $x_{e}$ of the two possible solutions.

Having determined the coordinates of points of the mechanism $\theta_{35}$ - the angle between links 3 and 5 is determined by using the scalar ratio of two vectors (4.8).

$$
l_{3} \cdot l_{5} \cdot \sin \theta_{35}=\left(x_{D}-x_{C}\right)\left(x_{E}-x_{C}\right)+\left(y_{D}-y_{C}\right)\left(y_{E}-y_{C}\right) \text {. }
$$

After the transformation Eq.(4.9) describing the value of the angle $\theta_{35}$ is obtained.

$$
\theta_{35}=\arcsin \frac{\left(x_{D}-x_{C}\right)\left(x_{E}-x_{C}\right)+\left(y_{D}-y_{C}\right)\left(y_{E}-y_{C}\right)}{l_{3} \cdot l_{5}} .
$$

The angle $\theta_{35}$ uniquely determines the angle of flexion of the knee of the rehabilitated person. Changing the angle depends on the inclination angle of the drive link $\left(\theta_{l}\right)$ and on the $X$ position of the fulcrum drive (point $A$ ).

Making changes of the coordinate $x_{a}$ affects the extreme values of the angle $\theta_{35}$. In this way, at a constant rotational speed of the drive link, the change of the $\theta_{35 \max }$ and $\theta_{35 \min }$ is possible.

\section{Computer program for analysis of the proposed CPM device}

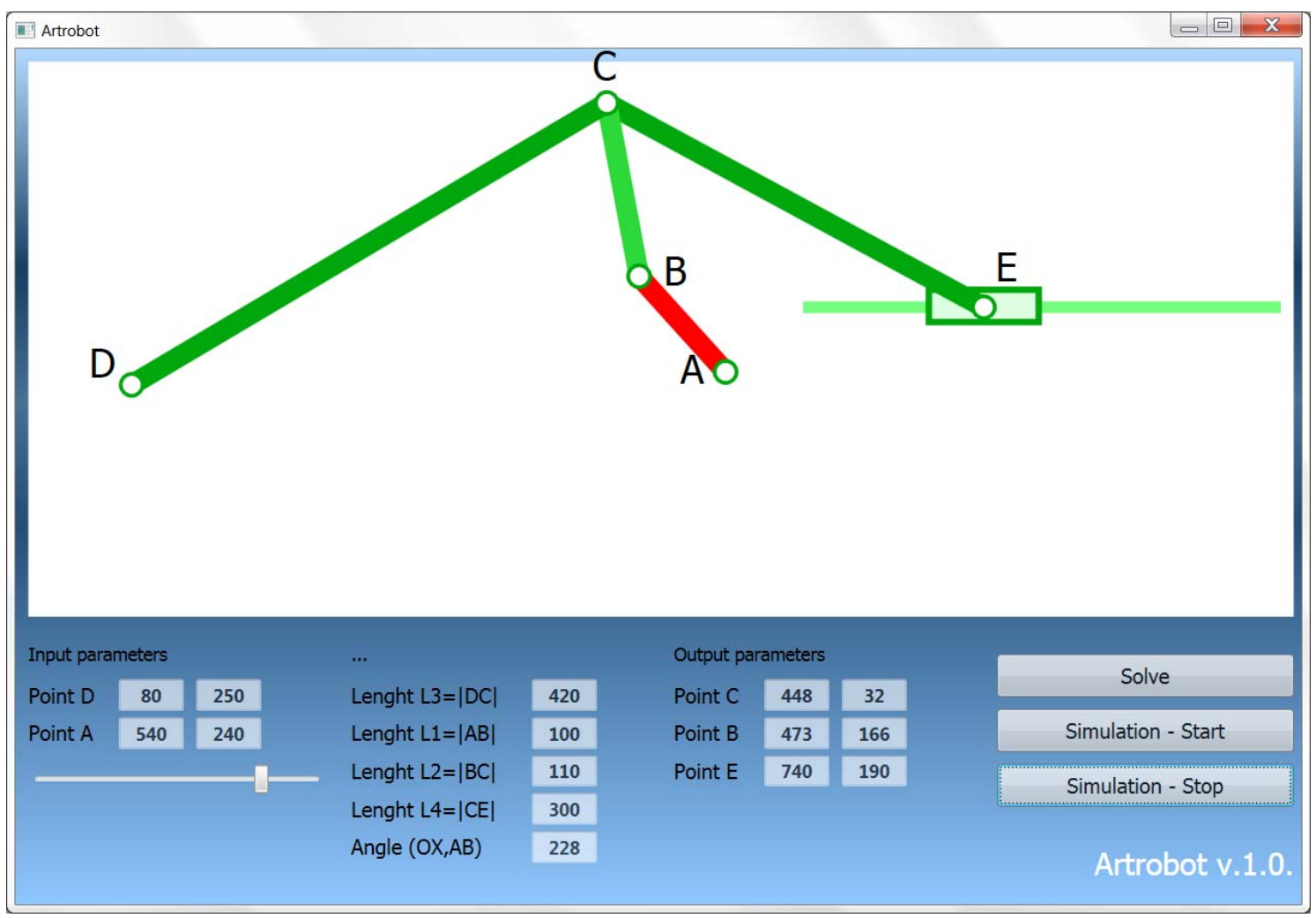

Fig.4. Main window of the application - "Artrobot". 
On the basis of this solution of the simple kinematics task computer algorithms are developed. Based on these algorithms an application is created and its main window is shown in Fig.4. The application was written in C\# with Microsoft Visual Studio 2012 using Windows Presentation Foundation library. The application allows you to simulate the operation of the mechanism. The application called "Artrobot" determines the motion parameters (position, velocity, acceleration) of the characteristic points of the mechanism.

The input data are the geometric parameters $l_{1}, l_{2}, l_{3}, l_{5}$ and the coordinates of points $x_{A}, y_{A}, x_{D}, y_{D}, y_{E}$. The program allows determination of the position of the mechanism at the set value $\theta_{I}$ - the angle of the drive link. It is also possible to run the simulation, wherein the drive link makes a full rotation (360 degrees). Then the application simulates the operation of the mechanism and presents it in motion. Successive positions of points, together with their velocities and accelerations are saved to an external file. This allows further data processing and analysis in other programs.

Using this application a simulation of the operation of the mechanism is carried out at different rotational velocity of the drive link. Angular range of changes $\theta_{35}$ at different positions of the fulcrum drive link (coordinate $X$ of the point $A$ ) is calculated.

In the proposed construction the drive link rotates continuously without changing the direction of rotation. This directly affects the fluidity of movement, durability and easier selection of the drive link.

\section{Conclusions and future works}

The method of rehabilitation with the use of the CPM device affects the acceleration of the process of post-operative knee rehabilitation after arthroscopy and arthroplasty.

It allows, among others, the improvement of metabolism within the joint, accelerates healing of damaged parts of the joint and reduces increased soft tissue tension. Furthermore, one can observe a faster absorption of hematoma, a better blood supply to the limb and increased strength of the ligament tension. The above-mentioned method improves inhibiting the clotting of blood and reduces discomfort during exercise (Wilk et al., 2004).

All things considered, one can undoubtedly say that work towards the development of new mechatronic solutions is legitimate.

Formulas presented in this paper describing the kinematics task of the proposed CPM device were used to create proprietary software that enables a numerical simulation of the mechanism. The application works with the assumed parameters describing the length of the individual links and their position in the global coordinate system.

The next phase of research will be related to the construction of the device. This software will allow a faster optimization of solutions, depending on the adopted initial conditions. The results of an analysis will be used to select a solution that will become the basis for the development of a solid model in the engineering environment, and then to build authors' own prototype device.

This research has been done as a part of a project No. S/WM/1/2012, which is funded by Bialystok Technical University, Poland.

\section{Nomenclature}

$$
\begin{aligned}
l_{1}, l_{2}, l_{3}, l_{5} & - \text { length of the links } \\
x_{A}, y_{A}, x_{B}, y_{B}, x_{C}, & - \text { the coordinates of points of the mechanism } \\
y_{C}, x_{D}, y_{D}, x_{E}, y_{E} & \\
\theta_{1} & - \text { the angle of the drive link } \\
\theta_{35} & - \text { the angle between } 3 \text { and } 5 \text { links }
\end{aligned}
$$




\section{References}

Artro-Med Kraków (9 May 2014) http://www.artro-med.pl/jace.html

http://panoramafirm.pl/małopolskie,,kraków,podgórze,saska,9_5/artro_med-abaclo_ror.html

Driscoll O., Shawn W. and Giori N.J. (2000): Continuous passive motion (CPM): theory and principles of clinical application. - Journal of Rehabilitation Research and Development, vol.37, No.2, pp.179-188.

Erhan A.G., Ertu` G.T., AA M (2009): Knee rehabilitation using an intelligent robotic system. - Journal of Intelligent Manufacturing, vol.20, No.2, 195-202.

Hunter Distributing (9 May 2014)

http://www.hunterdist.com/index.php?main_page=product_info\&cPath=344_366\&products_id=1314

Ortega A.B., Leyva J.L., Valdés G.V. and Conde E.C. (2012): Control of a knee rehabilitation machine using a virtual prototype. In Frontiers in Computer Education, pp.601-609, Springer, Berlin Heidelberg.

Rupp R., Plewa H., Hofer E.P. and Knestel M. (2009): MotionTherapy@ Home-A robotic device for automated locomotion therapy at home. In: Rehabilitation Robotics. - ICORR 2009. IEEE International Conference on. IEEE, pp.395-400.

Saringer J. (1993): Engineering aspect of the design and construction of continuous passive motion device for humans chapter in monography: Salter R.: Continuous Passive Motion: A Biological Concept for the Healing and Regeneration of Articular Cartilage, Ligaments, and Tendons: From Origination to Research to Clinical. Baltimore: Williams\&Wilkins.

Wilk M., Frańczuk B., Trąbka R. and Szwarczyk W. (2004): Preliminary results of early rehabilitation process of the patient with osteoarthritis of the knee arthroplasty using a continuous passive motion machine (in Polish). Fizjoterapia Polska, vol.4, No.2, pp.163-166.

Received: June 3, 2014

Revised: October 10, 2014 